\title{
Atypical hemolytic uremic syndrome in the Colombian Caribbean: its particular characteristics
}

\author{
Omar Cabarcas-Barbosa ${ }^{1,2}$. Gustavo Aroca-Martínez ${ }^{1,2}$. Carlos G. Musso ${ }^{1,6}$ (D) Elizabeth Ramos-Bolaños ${ }^{2,3}$. \\ Henry González-Tórres ${ }^{1,2}$. Zilac Espitaleta-Vergara ${ }^{1,2}$. Alex Domínguez-Vargas ${ }^{1}$. Edufamir Ararat-Rodriguez ${ }^{1}$. \\ José Orozco $^{1} \cdot$ Luis Castillo-Parodi $^{1,2}$. Juan Conde-Manotas ${ }^{1,3} \cdot$ Rodrigo Daza-Arnedo $^{1,4}$. \\ Víctor Rodríguez-SanJuan ${ }^{1} \cdot$ Liliana Gómez-Navarro $^{7} \cdot$ Roberto Acosta-Madiedo $^{8}$. Luis Barros-Camargo ${ }^{5}$. \\ Angélica Aduen-Carrillo ${ }^{3}$. Francisco Ayola-Anaya ${ }^{3} \cdot$ María Pulgar-Emiliani $^{1} \cdot$ Andrés Cadena-Bonfanti $^{1,2}$
}

Received: 19 September 2020 / Accepted: 4 October 2021 / Published online: 13 October 2021

(c) The Author(s), under exclusive licence to Springer Nature B.V. 2021

\begin{abstract}
Introduction Atypical hemolytic uremic syndrome (aHUS) is a rare and genetically mediated systemic disease most often caused by uncontrolled and chronic complement activation that leads to systemic thrombotic microangiopathy, renal and extra-renal damage.

Materials and methods This is descriptive, retrospective and multicenter study, which reports demographic, clinical, laboratory, and genetic characteristics, as well as their treatment response and outcome of 20 aHUS patients diagnosed between 2014 and 2018.

Results Most patients were female adults (75\%) and 30\% were associated to pregnancy/postpartum, $15 \%$ to autoimmune disease, and $65 \%$ to infections. Gastrointestinal involvement (75\%) was the most frequent extra-renal organ damage. Antenatal mortality and mortality rate were $5 \%$ and $10 \%$, respectively. $25 \%$ of the patients progressed to end-stage renal disease. In $4 / 8$ of patients treated within 1 week of presentation, eculizumab treatment restored multi-organ function after 4 weeks of treatment. CFH (37\%) and CFI (25\%) mutations were the most frequent.

Conclusion This is the first series of aHUS cases of Colombian Caribbean region which reports the clinical and epidemiological characteristics of this condition in this region.
\end{abstract}

Keywords Atypical hemolytic uremic syndrome $\cdot$ Thrombotic microangiopathy $\cdot$ Extra-renal manifestations

Carlos G. Musso

carlos.musso@hospitalitaliano.org.ar

1 Facultad de Ciencias de Ciencias de la Salud, Universidad Simón Bolívar, Barranquilla, Colombia

2 Clínica de la Costa, Barranquilla, Colombia

3 Clínica San Rafael, Sabanalarga, Colombia

4 Servicio de Cuidados Intensivos Obstétricos, Hospital Niño Jesús, Barranquilla, Colombia

5 Fresenius Medical Care, Barranquilla, Colombia

6 Unidad de Biología Del Envejecimiento, Hospital Italiano de Buenos Aires, Buenos Aires, Argentina

7 Unidad Renal Davita, Barranquilla, Colombia

8 Servicio Nefrología, Clínica Centro, Barranquilla, Colombia

\section{Introduction}

Hemolytic uremic syndrome (HUS), characterized by the triad of microangiopathic hemolytic anemia, thrombocytopenia and acute kidney injury (AKI), is a microvascular occlusion disorder that belongs to the category of thrombotic microangiopathy (TMA) $[1,2]$.

HUS may present primarily or due to secondary causes, such as bone marrow transplant, medication (e.g., anticancer chemotherapy, calcineurin inhibitors and antiplatelet agents), pregnancy or delivery, malignant hypertension, pneumococcal or viral infections and autoimmune diseases. In the case of primary HUS, the atypical form (aHUS) is distinguished from the typical form by the absence of a prior verotoxin-producing Escherichia coli infection. aHUS is most often caused by chronic, uncontrolled activation of the complement system, which leads to activation of endothelial 
cells, recruitment of platelets and TMA [1-3]. Regardless of the aHUS etiology, patients suffering from this condition may have extra-renal manifestations (e.g., neurological, cardiovascular, pulmonary and gastrointestinal), and poor outcomes: up to $25 \%$ of mortality rate, and about $50 \%$ of kidney disease progression to end-stage renal disease (ESRD) [2-4]. In this sense, a French cohort of 214 aHUS patients showed that $56 \%$ of them progressed to ESRD or died within the first year of follow-up [5].

Genetic or acquired dysregulation of the complement alternative pathway is detected in 40-60\% of patients with aHUS, suggesting a genetic predisposition $[4,5]$. This dysregulation is caused by mutations in genes that encode complement regulatory proteins: Factor $\mathrm{H}(\mathrm{FH})$, Factor I (FI), membrane cofactor protein (MCP), complement 3 (C3), Factor B (FB) or thrombomodulin (THBD) as well as in their combinations, or presence of anti-FH antibody resulting in activation of the complement system [6]. In addition, common single-nucleotide polymorphisms (SNPs) or haplotypes in $C F H, C F H R l$, and $M C P$ genes are risk factors for aHUS [7-11]. Large patient registries are required to characterize the natural history of this disease and to evaluate the impact of different therapies on it. The aHUS patients' genetic screening from registries has provided an estimation of the mutation frequency in the complement genes $[4,12$, 13]. In contrast, our knowledge of the aHUS presentation and outcome in our region is limited. Therefore, the aim of this study was to describe a series of aHUS cases in adult patients from the Colombian Caribbean region.

\section{Materials and methods}

This is the first descriptive, retrospective, and multicenter study which included adult patients with aHUS diagnosed during 2014-2018 from the Colombian Caribbean region. The aHUS clinical diagnosis was based on the presence of three criteria, as follows [3, 14]: (a) microangiopathic hemolytic anemia documented by the presence of schistocytes $>2 \%$ on peripheral smear, haptoglobin level $<30 \mathrm{mg}$ / $\mathrm{dL}$ (normal range 30-200 $\mathrm{mg} / \mathrm{dL}$ ), lactate dehydrogenase $(\mathrm{LDH})>500 \mathrm{U} / \mathrm{L}$ (normal range $250-500 \mathrm{U} / \mathrm{L}$ ), negative Coomb's test, normal ADAMTS13 activity, negative serologic testing, and stool, blood and urine cultures for Shigatoxin-producing E. coli or Shigella dysenteriae. (b) AKI defined as serum creatinine increase $>0.3 \mathrm{mg} / \mathrm{dL}$ within $48 \mathrm{~h}$ in healthy kidney patients (or creatininemia increase $>1.5$ times within 7 days in chronic nephropathy patients), and/ or urinary output reduction $<0.5 \mathrm{~mL} / \mathrm{h}$ for $6 \mathrm{~h}$ (c) Thrombocytopenia (platelet count $<150 \times 10^{3} / \mathrm{mm}^{3}$ or reduction of $>25 \%$ of previous platelet count).

Finally, renal biopsy was just performed in three aHUS patients who were suspected of having another renal disease, and of course, thrombotic microangiopathy was histologically confirmed in these patients.

In addition, all cases of aHUS secondary to hematopoietic stem cell or solid-organ transplantation, malignancy, drugs, and with ADAMTS13 activity $\leq 5 \%$ (the level consistent with thrombotic thrombocytopenic purpura diagnosis) were excluded.

Besides, patients included on the sample were not required to have an identified complement gene mutation or factor $\mathrm{H}$ autoantibody, or prior or ongoing eculizumab treatment. Study data were obtained from the medical records of patients with aHUS from the participating medical centers. Relevant clinical and complementary studies data were collected: patient's main demographic and medical antecedents, autoimmune-related diseases, extra-renal clinical manifestations, renal ultrasound, serum and urine biochemical parameters: hemogram, haptoglobin, lactate dehydrogenase (LDH), schistocytes in peripheral blood smear, total bilirubin, aspartate amino transferase (AST), alanine transaminase (ALT), arterial blood gases, serum potassium, serum bicarbonate, urinalysis, 24-h proteinuria, C3-C4 complement, and ADAMTS13 value, associated treatments and concomitant medications, reported clinical outcomes, and genetic test (if available).

All patients were vaccinated against Neisseria meningitides prior to eculizumab initiation and received prophylactic antibiotics up to 2 weeks post-eculizumab [15]. The categorical variables were described through absolute and relative frequencies. Range, mean and standard deviation were used to analyze the continuous variables. Data were stored in a MySQL ${ }^{\circledR}$ database and it was exported to R-CRAN statistical software [16], in which the analysis was performed.

Informed consent was obtained from all individual participants included in the study and this study was approved by the Ethics Committee of the Clínica de la Costa de Barranquilla, Colombia.

\section{Results}

Twenty patients suffering from aHUS were diagnosed during the study period. Most patients were female (15/20), with a mean age of 39 years (range 23-62 years). Six (30\%) patients had aHUS related to pregnancy/postpartum: $2 / 6(10 \%)$ were pregnant for the first time and $4 / 6(20 \%)$ had had a previous normal delivery. Obstetric aHUS appears from 24 weeks of pregnancy to 8 days of postpartum, with $1 / 6,3 / 6$, and $2 / 6$ patients presenting obstetric aHUS in second trimester (13-27 weeks), third trimester (28-week delivery) and postpartum, respectively (Table 1). Five pregnancies $(25 \%)$ resulted in live births and $1(5 \%)$ resulted in fetal death in the third quarter of 


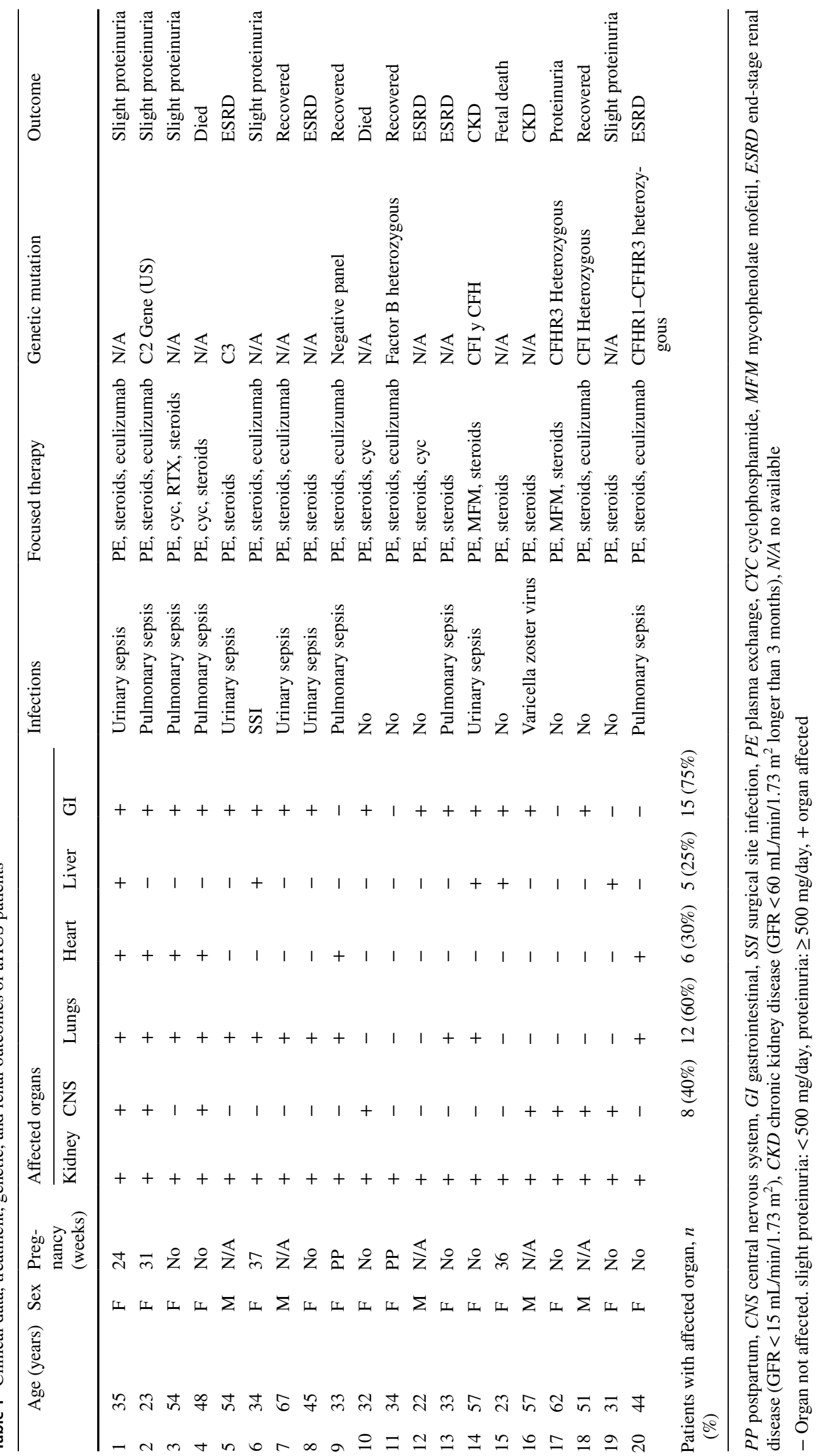


pregnancy. Three babies were premature (26-, 28- and 33-week gestation) after emergency cesarean section due to severe preeclampsia resulting in premature delivery within $96 \mathrm{~h}$ of aHUS appearance. Three (15\%) patients presented aHUS related to autoimmune disease: $2(10 \%)$ had systemic lupus erythematous, and 1 (5\%) had scleroderma, while 13 cases $(65 \%)$ were associated to infection, being pulmonary sepsis the most frequent one (30\%) (Table 1). Regarding the main hematologic parameters, hemoglobin $(\mathrm{Hb})$ levels were low in all patients: mean $\mathrm{Hb} 8.2 \mathrm{~g} / \mathrm{dL}$ (range $5.4-12.1 \mathrm{~g} / \mathrm{L}$ ), as well as mean platelets count, which was $67 \times 10^{3} / \mathrm{mm}^{3}$ (range $9-140 \times 10^{3}$ ) $\mathrm{mm}^{3}$ ). Besides, low serum C3 levels were observed in 12 cases (60\%): mean $84.2 \mathrm{mg} / \mathrm{dL}$ (range $45-150 \mathrm{mg} / \mathrm{dL}$ ). The rest of the main laboratory parameters of the aHUS patients are outlined in Table 2 . In regards to the renal manifestations, 15 patients ( $75 \%$ ) presented hypertension, 12 of them were on angiotensin-converting enzyme inhibitor (ACEI) or angiotensin II receptor blocker (ARA), in combination with beta-blocker in 2 patients, and with calcium channel blocker in 2 patients, and 3 patients were on methyldopa. Eighteen patients (90\%) presented several degrees of AKI. Nine patients (45\%) showed oliguria or anuria, and required hemodialysis sessions for up to 5 days (cases $4,5,8,10,12,13,14,16$ and 20). AKI was related to high serum potassium levels in $40 \%$ ( $n$ : 8) of patients (serum potassium $5.3 \pm 0.9 \mathrm{mmol} / \mathrm{L}$, range $3.6-6.3 \mathrm{mmol} / \mathrm{L}$ in the study group), as well as metabolic acidosis in 50\% ( $n$ : 10) of patients (serum pH: $7.30 \pm 0.3$, range 6.5-7.4, and serum bicarbonate $19.7 \pm 3.9 \mathrm{mmol} / \mathrm{L}$, range $12.6-26 \mathrm{mmol} / \mathrm{L}$, in the study group). In addition, 10 patients (50\%) had proteinuria, and 8 (40\%) had microscopic hematuria in spot urine samples. Renal ultrasounds were informed as normal, except for one who showed signs of acute nephropathy, and three presented renal changes of chronicity. It is worth mentioning that an additional glomerular pattern was found in three aHUs patients (15\%) in renal biopsy, as follows: one case had a membranoproliferative glomerulonephritis pattern associated with fibrinoid thrombi and vasculopathy (case 13). The second case had a diffuse proliferative lupus nephritis (class IV-G), activity and chronic index was $14 / 24$ and $2 / 12$, respectively (case 10). Lastly, the third case had a pauciimmune rapidly progressive glomerulonephritis associated to ANCA (case 5). All patients experienced extra-renal manifestations as outlined in Table 1 and most experienced multiple organ failure. Seventeen patients (85\%) had $\geq 2$ extra-renal manifestation and 4 patients $(15 \%)$ had $\geq 4$ extra-renal manifestations (Table 1). Concerning the gastrointestinal involvement, it was the most frequent one $(n=15,75 \%): 10$ patients $(50 \%)$ had abdominal pain, $2(10 \%)$ diarrhea, and $5(25 \%)$ gastrointestinal bleeding (melena or hematochezia). Liver injury was present in 5 patients (25\%), and 3 patients (15\%) had jaundice with high mixed serum bilirubin levels.

With respect to patients' respiratory symptoms, 12 (60) presented dyspnea, 5 were associated to acute respiratory distress syndrome (ARDS), while 4 were associated to acute pulmonary edema.

Finally, CNS involvement $(n=8,40 \%)$ included loss of consciousness $(25 \%)$ and generalized seizures $(15 \%)$. Ischemic or hemorrhagic cerebrovascular disease was present in 2 cases (15\%) with pregnancy-associated aHUS. Cardiac involvement $(n=6,30 \%)$ mainly manifested as acute cardiac failure with dilated cardiac chambers and decreased ejection fraction (minimum 22\%). One patient had a noncoronary acute myocardial infarction, since absence of coronary artery lesions was confirmed by coronary angiogram.

Concerning to aHUS treatment and outcomes, an overview of them is outlined in Table 1. Plasma therapy, both small volume infusion of fresh-frozen plasma $(n=15$, $75 \%)$ and plasmapheresis $(n=20,100 \%)$, was initiated in all patients. In addition, some patients received immunosuppressant (cyclophosphamide or mycophenolate), low molecular weight heparin, antibacterial, and dialysis therapy). Twelve patients were on antiproteinuric agents, such as ACEI or ARA. Regarding eculizumab treatment, it induced clinical and laboratory normalization, and full reversal of TMA manifestations with restoration of renal and extra-renal functions after 4 weeks of its initiation in $4 / 8$ patients treated within 1 week of presentation (Table 1). Eculizumab was discontinued following the induction phase in two patients who achieved clinical remission (renal and hematologic) as local availability was restricted. Despite initial decrease in the severity of renal and extra-renal manifestations after the first two infusions of the drug, $3 / 8$ patients whose eculizumab initiation occurred at least 3 weeks postpresentation remained with slight proteinuria ( $>500 \mathrm{mg} /$ day) being on ACEI or ARA (Table 1). No side effects were observed with eculizumab treatment in this study. Heterozygous mutations in CFHR1-CFHR3 were detected in $1 / 8$ genetic screening available (Table 1). This patient received eculizumab 1 month after initial disease presentation and reached complete remission of TMA and extra-renal aHUS manifestations. However, kidney function was not restored, ESRD developed possibly due to delayed initiation of eculizumab treatment. Antenatal mortality and mortality rate were 5\% and $10 \%$, respectively. Patients who died had higher LDH levels (1344 versus $1222 \mathrm{U} / \mathrm{L}$ ) and more severe thrombocytopenia $\left(12\right.$ versus $7510^{3} / \mathrm{mm}^{3}$ ) than survivors. Five patients (25\%) progressed to ESRD (Table 1). Genetic screening to assess alterations in complement regulatory proteins was available in $8 / 20(40 \%)$ patients as outlined in Table 1. CFH $3 / 8$ (37\%) and CFI 2/8 (25\%) mutations were the most frequent. One case reported $\mathrm{C} 2$ gene mutation as an alteration of uncertain significance for aHUS [13]. 


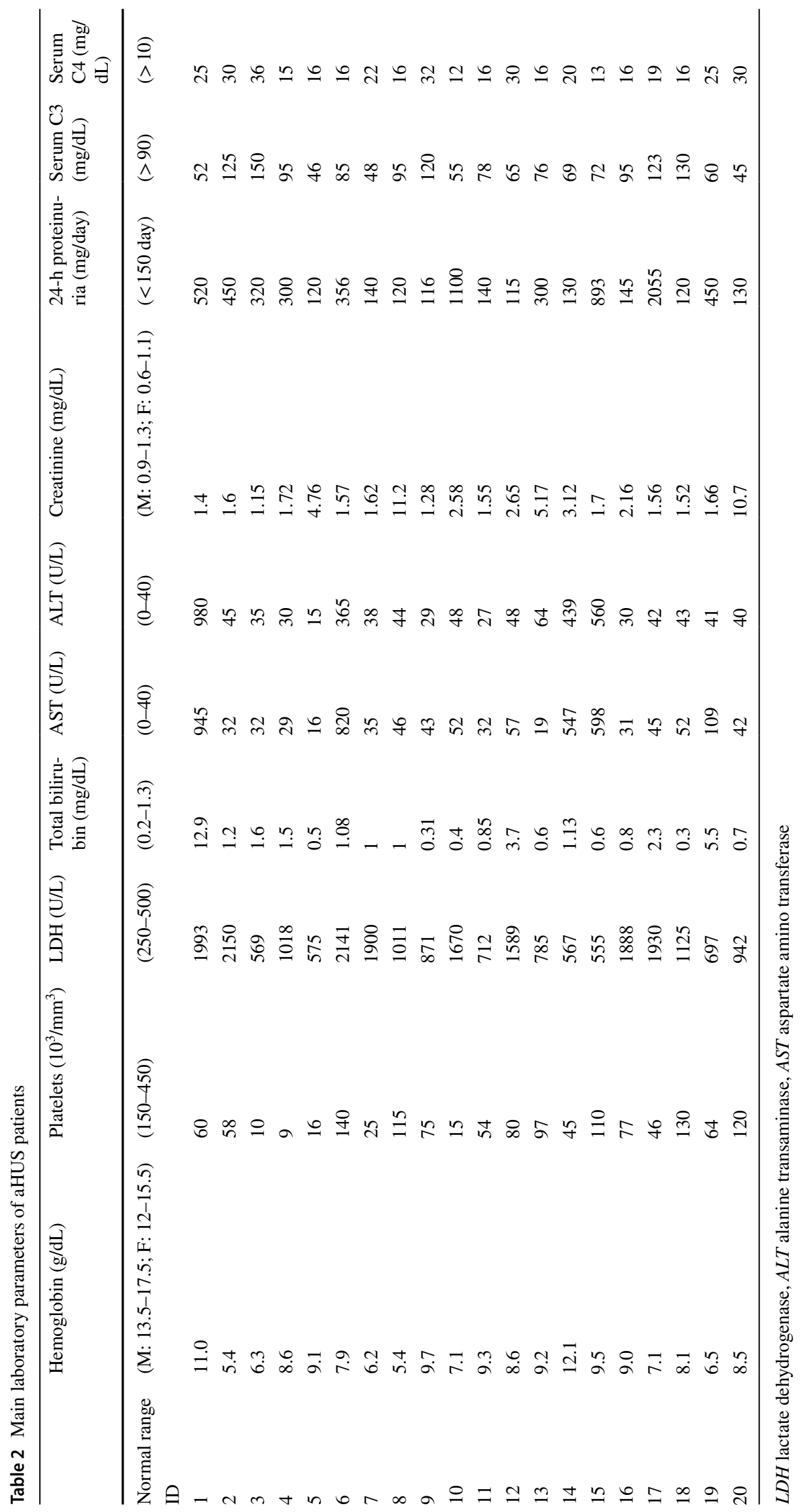




\section{Discussion}

This study describes a series of 20 adult cases with aHUS from the Colombian Caribbean region. Since all the evaluated patients had a preserved ADAMTS13, thrombotic thrombocytopenic purpura was ruled out as the cause of their TMA [1]. Some conditions are known to amplify complement and unmask latent complement defects resulting in aHUS manifestation in predisposed individuals. These potential precipitating factors include autoimmune diseases, transplants, infections, drugs, and metabolic conditions $[2,3,6]$. In the present study, $3(15 \%)$ cases had aHUS autoimmune-related disease and $13(65 \%)$ had bacterial or viral infection. Pregnancy is also a powerful complement activator, accounting for 7\% of aHUS cases [17]. In this report, six (30\%) had aHUS related to pregnancy/ postpartum: $2 / 6(10 \%)$ were pregnant for the first time and $4 / 6(20 \%)$ had previously given birth to healthy children. Under physiological conditions, complement activation in pregnancy is regulated by three proteins which are present in the trophoblast membrane: decay-accelerating factor (DAF), membrane cofactor protein (MCP), and CD59. When complement system activation exceeds the regulatory ability of the previously mentioned proteins, injury is produced specially in the endothelial cells of human placenta. This phenomenon leads to the potential risk of premature deliveries and fetal loss, as observed in the present study $[4,18,19]$.

Following delivery, mothers lose these placental protective mechanisms. This and the presence of inflammation, an extensively wounded uterus, influx of fetal cells into maternal blood, bleeding, and infection can lead to the systemic activation of the alternative complement pathway, and postpartum aHUS [18, 20]. Evidence of microangiopathic hemolytic anemia should be confirmed with a peripheral blood smear showing schistocytes and helmet cells $[2,17]$. However, in this study, two patients showed no schistocytes in peripheral blood smear. This phenomenon could be explained since there are patients with intact reticuloendothelial system, which has the ability to rapidly remove peripheral blood schistocytes.

In addition, no significant alteration in serum transaminases, bilirubin levels, and haptoglobin levels were documented. Even though these values were not the theoretically expected ones in a setting of hemolysis, they represent the balance between production and removal of these substances, and not the presence or absence of hemolysis; thus, they could actually have any serum value [10]. Therefore, it is recommended to perform a serial sample on peripheral blood smear, and to be interpreted by an expert in hematologic cytopathology to confirm the presence of fragmented red blood cells [1,21, 22]. Other laboratory parameters consistent with hemolytic anemia are an elevated serum LDH level and a low serum haptoglobin concentration $[17,23]$. In addition, Coombs test should be performed to rule out the presence of an autoimmune hemolytic anemia, therefore, being negative in cases of aHUS [9, 23, 24]. Kidney biopsy is not considered necessary to diagnose primary TMA, although it can be useful when secondary aHUS is suspected $[3,23]$. In this study, biopsy was performed in patients with active systemic lupus erythematous or other suspected glomerular disease. A membranoproliferative glomerulonephritis pattern, a diffuse proliferative lupus nephritis (class IV-G) and a pauci-immune rapidly progressive glomerulonephritis associated to ANCA were detected. There is an established correlation between aHUS and membranoproliferative glomerulonephritis (C3 glomerulopathy) whose physiopathology involves genetic alterations in the regulatory proteins of the alternative complement pathway. Based on this finding, it has been hypothesized that both conditions could be the same entity with different phenotypes, varying from aHUS to $\mathrm{C} 3$ glomerulopathy (DDD or C3 glomerulonephritis) [4, 14, 23, 25]. Hypocomplementemia was seen in $60 \%$ of the cases; however, this finding is not very sensitive or specific, being reported in only $30-50 \%$ of cases [25]. Fakhouri et al. reported $85 \%$ of cases with disorders in the complement regulatory proteins, being CFI mutation frequently documented [18]. Huertas et al. [26] reported in his study cohort genetic disorders in $41 \%$ of cases, notably without MCP protein and anti-HF antibody mutations. Despite aHUS diagnosis is mainly clinic, and its treatment initiation does not depend on any genetic mutation identification, its presence should be ruled out because of its influence on nephropathy progression to ESRD, and the aHUS recurrence risk in kidney transplantation [2, 4, 23, 24]. In the present study, the different aHUS inducing identified mutations are detailed in Table 1. Because of the high morbidity and mortality associated with aHUS, it is crucial to have an understanding of the potential clinical manifestations of the disease and its associated laboratory findings to ensure prompt diagnosis and treatment [17]. aHUS typically is characterized by renal involvement [24]; however, medical literature is increasingly reporting extra-renal complications of the disease, including cardiovascular, neurological, pulmonary, and gastrointestinal [4, 19, 27]. Gastrointestinal complications of HUS are quite exclusive of Shiga-toxin-producing E. coli-associated HUS, whereas aHUS have usually mild or absent intestinal involvement [28]. In our study, gastrointestinal involvement $(n=15$, $75 \%$ ) was the most frequent extra-renal manifestation. Recent studies have evidenced that gastrointestinal complications and symptoms, as well as pancreatitis, are more common in aHUS with anti-factor-H autoantibodies [3, 
$17,28]$. Diarrhea is also observed in approximately $50 \%$ of patients preceding or during the acute phase of aHUS [17]. Thus, a further anti-factor-H autoantibodies assessment is required to identify a possible association in our population. Neurological manifestations (seizures, confusion, diplopia, blindness, hemiparesis, etc.) are usually described in $20-48 \%$ of the cases in the literature [21]. In the present investigation, it was documented that 11 patients $(55 \%)$ presented some neurological manifestation, from altered state of consciousness, seizures to ischemic/ hemorrhagic stroke. Cerebrovascular events occurred in three pregnancy-associated aHUS cases. Fakhouri et al. reported 4 patients $(18 \%)$ with neurological manifestations in a cohort of 22 cases with pregnancy-associated aHUS [18]. In a collaborative work between the United Kingdom and Italy, Bruel et al. [29] described neurological involvement in 7 out of 87 cases (8\%), similarly to another study published by Huertas et al. [26] describing 7 cases of neurological involvement. However, it should be pointed out that the study performed by Huerta et al. [26] was a specific descriptive study of only patients with aHUS associated with pregnancy, where 22 cases were studied, with 7 patients with neurological symptoms (7/22:31.8\%). On the other hand, in the present study, only six were associated with pregnancy, and two cases had neurological symptoms (2/6: 33\%). Therefore, the frequency of neurological symptoms in our cases with pregnancy-associated aHUS was in fact similar to that reported by Huerta et al. [26]. Even more, the frequency of neurological symptoms was similar in this study to that reported in the literature, if all the reported patients are taken into account $[6,9,21]$. Thus, it seems that our population did not present more neurological compromise compared to other published reports. Pulmonary complications of aHUS are typically seen in cases of multiple organ dysfunction and pulmonary edema associated with cardiac dysfunction and/or systemic volume overload [17]. Bruel et al. [29] reported three cases, two had acute pulmonary edema, and one had pulmonary thromboembolism. In our study, progressive respiratory failure occurred in 12 patients due to pulmonary edema caused by acute respiratory distress syndrome or pulmonary sepsis. Most of the patients developed and required hemodialysis (59\% of children and $81 \%$ of adults). Other manifestations of aHUS induced renal damage, aside from an elevated serum creatinine, were the presence of hematuria, proteinuria, and edema $[3,6,30]$. Kidney involvement, defined by an elevated serum creatinine, presence of dysmorphic hematuria, proteinuria, or/and altered renal imaging, occurred in all the studied patients. Those aHUS patients who required dialysis were the ones whose clinical presentation was a severe acute kidney injury. Clinical outcomes in aHUS were historically poor: over half of patients managed with plasma sustained permanent renal damage, progressed to ESRD, or died within 1 year of diagnosis [17]. Eculizumab, a monoclonal humanized antibody directed against $\mathrm{C} 5$, is the mainstay of treatment for aHUS [10], and it was initiated within 1 week of aHUS diagnosis in 4/8 patients. Hematological signs normalized and the function of damaged organs, including the kidneys, brain, and lungs, was fully restored after 4 weeks of treatment initiation. In those $3 / 8$ cases whose eculizumab initiation occurred at least 3 weeks postpresentation remained with slight proteinuria. When eculizumab treatment was started later (1-month post-diagnosis) the outcomes in 1/8 case were ultimately unfavorable resulting in ESRD. Even our findings demonstrated the benefit of early treatment of aHUS with eculizumab, still it has certain limitations, the main one being the lack of available genetic screening, which would identify mutations in the genes of the complement system. However, identification of a complement mutation or anti-CFH inhibitory antibody is not required to make aHUS diagnosis nor to initiate therapy, still it does inform on prognosis and risk of recurrence.

\section{Conclusion}

This is the first series of atypical hemolytic uremic syndrome (aHUS) cases of Colombian Caribbean region being reported, and this study documented that aHUS in this region presented the classical clinical and epidemiological characteristics which characterize this condition, even presenting similar extra-renal manifestations than previously reported cases in the literature. This report also reinforces the concept that the delay in early diagnosis and effective treatment of this condition can lead to poor outcomes.

Funding No funds were received for performing this study.

\section{Declarations}

Conflict of interest All the authors declare that they have no conflict of interest.

Ethical approval All the procedures performed in studies involving human participants were in accordance with the ethical standards of the institutional and/or national research committee and with the 1964 Helsinki Declaration and its later amendments or comparable ethical standards.

Informed consent Informed consent was obtained from all the participants included in the study.

\section{References}

1. Zipfel PF, Heinen S, Skerka C (2010) Thrombotic microangiopathies: new insights and new challenges. Curr Opin Nephrol 
Hypertens 19(4):372-378. https://doi.org/10.1097/MNH.0b013 e32833aff4a

2. Noris M, Remuzzi G (2009) Atypical hemolytic-uremic syndrome. N Engl J Med 361(17):1676-1687. https://doi.org/10. 1056/NEJMra0902814

3. Raina R, Krishnappa V, Blaha T et al (2019) Atypical hemolyticuremic syndrome: an update on pathophysiology, diagnosis, and treatment. Ther Apher Dial 23(1):4-21. https://doi.org/10.1111/ 1744-9987.12763

4. Noris M, Caprioli J, Bresin E et al (2010) Relative role of genetic complement abnormalities in sporadic and familial aHUS and their impact on clinical phenotype. Clin J Am Soc Nephrol 5(10):1844-1859. https://doi.org/10.2215/CJN.02210310

5. Fremeaux-Bacchi V, Fakhouri F, Garnier A et al (2013) Genetics and outcome of atypical hemolytic uremic syndrome: a nationwide French series comparing children and adults. Clin J Am Soc Nephrol 8(4):554-562. https://doi.org/10.2215/CJN.04760512

6. Fakhouri F, Zuber J, Frémeaux-Bacchi V, Loirat C (2017) Haemolytic uraemic syndrome. Lancet (London, England) 390(10095):681-696. https://doi.org/10.1016/S0140-6736(17) 30062-4

7. Fremeaux-Bacchi V, Kemp EJ, Goodship JA et al (2005) The development of atypical haemolytic-uraemic syndrome is influenced by susceptibility factors in factor $\mathrm{H}$ and membrane cofactor protein: evidence from two independent cohorts. J Med Genet 42(11):852-856. https://doi.org/10.1136/jmg.2005.030783

8. Ermini L, Goodship THJ, Strain L et al (2012) Common genetic variants in complement genes other than $\mathrm{CFH}, \mathrm{CD} 46$ and the CFHRs are not associated with aHUS. Mol Immunol 49(4):640648. https://doi.org/10.1016/j.molimm.2011.11.003

9. Campistol JM, Arias M, Ariceta G et al (2013) An update for atypical haemolytic uraemic syndrome: diagnosis and treatment. A consensus document. Nefrologia 33(1):27-45. https://doi.org/ 10.3265/Nefrologia.pre2012.Nov.11781

10. Picard C, Burtey S, Bornet C, Curti C, Montana M, Vanelle P (2015) Pathophysiology and treatment of typical and atypical hemolytic uremic syndrome. Pathol Biol 63(3):136-143. https:// doi.org/10.1016/j.patbio.2015.03.001

11. Licht C, Ardissino G, Ariceta G et al (2015) The global aHUS registry: methodology and initial patient characteristics. BMC Nephrol 16(1):207. https://doi.org/10.1186/s12882-015-0195-1

12. Sullivan M, Rybicki LA, Winter A et al (2011) Age-related penetrance of hereditary atypical hemolytic uremic syndrome. Ann Hum Genet 75(6):639-647. https://doi.org/10.1111/j.1469-1809. 2011.00671.x

13. Maga TK, Nishimura CJ, Weaver AE, Frees KL, Smith RJH (2010) Mutations in alternative pathway complement proteins in American patients with atypical hemolytic uremic syndrome. Hum Mutat 31(6):1445-1460. https://doi.org/10.1002/humu. 21256

14. Manenti L, Gnappi E, Vaglio A et al (2013) Atypical haemolytic uraemic syndrome with underlying glomerulopathies. A case series and a review of the literature. Nephrol Dial Transplant 28(9):2246-2259. https://doi.org/10.1093/ndt/gft220

15. European Medicines Agency (2016) Soliris [summary Prod Charact Alexion Eur SAS, (Paris, France)

16. R Core Team (2014) R: A Language and Environment for Statistical Computing. Published online
17. Formeck C, Swiatecka-Urban A (2019) Extra-renal manifestations of atypical hemolytic uremic syndrome. Pediatr Nephrol 34(8):1337-1348. https://doi.org/10.1007/s00467-018-4039-7

18. Fakhouri F, Roumenina L, Provot F et al (2010) Pregnancy-associated hemolytic uremic syndrome revisited in the era of complement gene mutations. J Am Soc Nephrol 21(5):859-867. https:// doi.org/10.1681/ASN.2009070706

19. Kozlovskaya NL, Korotchaeva YV, Bobrova LA (2019) Adverse outcomes in obstetric-atypical haemolytic uraemic syndrome: a case series analysis. J Matern Neonatal Med 32(17):2853-2859. https://doi.org/10.1080/14767058.2018.1450381

20. Gupta M, Govindappagari S, Burwick RM (2020) Pregnancyassociated atypical hemolytic uremic syndrome: a systematic review. Obstet Gynecol 135(1):46-58. https://doi.org/10.1097/ AOG.0000000000003554

21. Pablo Córdoba J, Mariel Contreras K, Larrarte C et al (2015) Síndrome hemolítico urémico atípico, revisión de la literatura y documento de consenso. Enfoque diagnóstico y tratamiento. Rev Colomb Nefrol 2(1):19-40

22. Schaefer F, Ardissino G, Ariceta G et al (2018) Clinical and genetic predictors of atypical hemolytic uremic syndrome phenotype and outcome. Kidney Int 94(2):408-418. https://doi.org/ 10.1016/j.kint.2018.02.029

23. Bajracharya P, Jain A, Baracco R, Mattoo TK, Kapur G (2016) Atypical hemolytic uremic syndrome: a clinical conundrum. Pediatr Nephrol 31(10):1615-1624. https://doi.org/10.1007/ s00467-016-3369-6

24. Loirat C, Fremeaux-Bacchi V (2011) Atypical hemolytic uremic syndrome. Orphanet J Rare Dis 6(1750-1172 (Electronic)):60. https://doi.org/10.1111/j.1442-200X.1995.tb03393.x

25. Zuckerman R, Asif A, Costanzo EJ, Vachharajani T (2018) Complement activation in atypical hemolytic uremic syndrome and scleroderma renal crisis: a critical analysis of pathophysiology. J Bras Nefrol 40(1):77-81. https://doi.org/10.1590/ 2175-8239-JBN-3807

26. Huerta A, Arjona E, Portoles J et al (2018) A retrospective study of pregnancy-associated atypical hemolytic uremic syndrome. Kidney Int 93(2):450-459. https://doi.org/10.1016/j.kint.2017. 06.022

27. Noris M, Remuzzi G (2014) Cardiovascular complications in atypical haemolytic uraemic syndrome. Nat Rev Nephrol 10(3):174180. https://doi.org/10.1038/nrneph.2013.280

28. Bianchi L, Gaiani F, Vincenzi F et al (2018) Hemolytic uremic syndrome: differential diagnosis with the onset of inflammatory bowel diseases. Acta Biomed 89:153-157. https://doi.org/10. 23750/abm.v89i9-S.7911

29. Bruel A, Kavanagh D, Noris M et al (2017) Hemolytic uremic syndrome in pregnancy and postpartum. Clin J Am Soc Nephrol 12(8):1237-1247. https://doi.org/10.2215/CJN.00280117

30. Lee JM, Park YS, Lee JH et al (2015) Atypical hemolytic uremic syndrome: Korean pediatric series. Pediatr Int 57(3):431-438. https://doi.org/10.1111/ped.12549

Publisher's Note Springer Nature remains neutral with regard to jurisdictional claims in published maps and institutional affiliations. 